This item was submitted to Loughborough's Research Repository by the author.

Items in Figshare are protected by copyright, with all rights reserved, unless otherwise indicated.

\title{
An ankle joint model-based image-matching motion analysis technique
}

\section{PLEASE CITE THE PUBLISHED VERSION}

http://dx.doi.org/10.1016/j.gaitpost.2011.03.014

\section{PUBLISHER}

(C) Elsevier

\section{VERSION}

AM (Accepted Manuscript)

\section{PUBLISHER STATEMENT}

This work is made available according to the conditions of the Creative Commons Attribution-NonCommercialNoDerivatives 4.0 International (CC BY-NC-ND 4.0) licence. Full details of this licence are available at: https://creativecommons.org/licenses/by-nc-nd/4.0/

\section{LICENCE}

CC BY-NC-ND 4.0

\section{REPOSITORY RECORD}

Mok, Kam-Ming, Daniel Tik-Pui Fong, Tron Krosshaug, Aaron See-Long Hung, Patrick Shu-Hang Yung, and Kai-Ming Chan. 2019. "An Ankle Joint Model-based Image-matching Motion Analysis Technique". figshare. https://hdl.handle.net/2134/21282. 


\section{Elsevier Editorial System(tm) for Gait and Posture Manuscript Draft}

Manuscript Number: GAIPOS-D-10-00352R1

Title: An ankle joint model-based image-matching motion analysis technique

Article Type: Original Paper

Keywords: video analysis, ankle joint motion, injury biomechanics

Corresponding Author: Dr Daniel Tik-Pui Fong, PhD

Corresponding Author's Institution: The Chinese University of Hong Kong

First Author: Kam Ming Mok, M.Phil

Order of Authors: Kam Ming Mok, M.Phil; Daniel Tik-Pui Fong, PhD; Tron Krosshaug, Ph.D.; Aaron SeeLong Hung, B.Sc; Patrick Shu-Hang Yung, MBChB ; Kai-Ming Chan, MBBS

Abstract: This study presented a model-based image-matching (MBIM) motion analysis technique for ankle joint kinematic measurement. Five cadaveric below-hip specimens were manipulated through a full range of ankle joint motions in bare-foot and shoe-wearing conditions. The ankle motions were analyzed by bone-pin marker-based motion analysis and MBIM motion analysis techniques respectively. The root mean square errors of all angles of motion were less than 3 degrees. The average Intraclass Correlation Coefficients (ICCs) for the intra-rater reliability were greater than 0.928 and the average ICCs for the inter-rater reliability were greater than 0.948 for all ranges of motion. Excellent validity, intra-rater reliability and inter-rater reliability were achieved for the MBIM technique in both bare-foot and shoe-wearing conditions. The MBIM technique can therefore provide good estimates of ankle joint kinematics. 
Ms. Ref. No.: GAIPOS-D-10-00352

Title: An ankle joint model-based image-matching motion analysis technique

Editor of Gait \& Posture,

Dear Prof. Theologis,

Authors would like to express their sincere thanks for this revision opportunity. Model-Based Image-Matching (MBIM) technique is a newly developed motion analysis tool, comments from experts and researchers could contribute to its further developments. We are welcoming all comments and hope to share the MBIM technique through Gait and Posture.

Again, we would like to declare that each author were fully involved in the study and preparation of the manuscript and that the material within has not been and will not be submitted for publication elsewhere.

Corresponding author during review process: Mr. Kam-Ming MOK, Department of Orthopaedics and Traumatology, Prince of Wales Hospital, Faculty of Medicine, The Chinese University of Hong Kong, Hong Kong, China, kmmok@ ort.cuhk.edu.hk

Corresponding author if paper is accepted: Prof. Daniel Tik-Pui FONG, Department of Orthopaedics and Traumatology, Prince of Wales Hospital, Faculty of Medicine, The Chinese University of Hong Kong, Hong Kong, China, dfong@ ort.cuhk.edu.hk

Thank you for your attention and help!

Yours sincerely,

Kam-ming, MOK

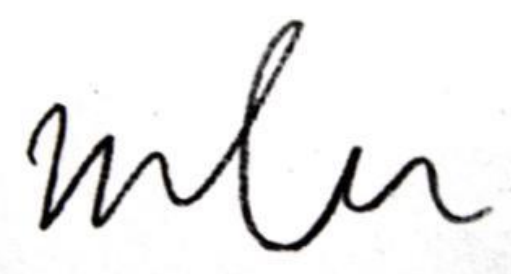

Kam Ming, MOK

2010.12.15 


\section{Conflict of Interest Statement}

Editor of Gait \& Posture,

REF: Submission of manuscript "An ankle joint model-based image-matching motion analysis technique"

The authors declare no financial and personal relationships with other people or organizations that could inappropriately influence this submitted work.

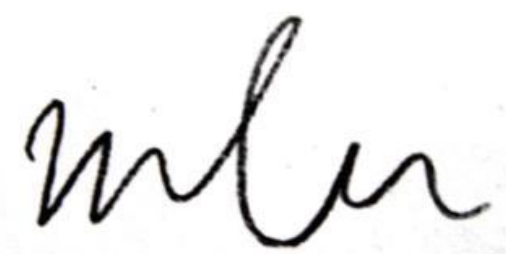

Kam Ming, MOK

2010.12.15 
Ms. Ref. No.: GAIPOS-D-10-00352

Title: An ankle joint model-based image-matching motion analysis technique Gait and Posture

\section{$\underline{\text { Re: Response to Reviewers }}$}

Reviewer \#1: This study looked to evaluate a Model Based Image Matching technique for evaluating ankle joint kinematics based on video data. Motion capture using retro-reflective markers was used as the gold standard. The goal was to establish reliability and validity of the measure.

Overall the study protocol is well defined to solve the suggested problem and the results have shown good reliability of the measurement. However there are areas within the manuscript that need greater explanation.

Introduction

In general the introduction is very sparse and needs further development. Specifically it is missing some key information such as literature on the cause of ankle sprains in general. Are the main factors kinematic in nature or kinetic, or a combination of the two? In which anatomical plane of motion do most ankle sprains occur?

Authors' response: Thank you for your comment. The whole introduction was rewritten as below.

Line 16-49:

"Ankle ligamentous sprain is one of the most common injuries encountered in sports (Fong et al., 2007; Fong et al., 2009a). A precise description of the injury situation is a key component to understanding the aetiology and injury mechanism (Bahr and Krosshaug, 2005). The injury mechanisms of ankle ligamentous sprain have been described as a combined inversion and internal rotation of the ankle joint (Safran et al., 1991), or plantarflexion with the subtalar joint adducting and inverting (Vitale \& Fallat, 1988). Fong et al. (2009b) reported the ankle joint kinematics from a single accidental ankle supination sprain case under skin-marker motion analysis, the finding is that dorsiflexion instead of plantarflexion was found at injury. A study analyzed the ankle supination sprain injuries using video analysis, Andersen et al. (2004) reported two major injury mechanisms as: (1) impact by opponent on the medial aspect of the leg just before or at foot strike, which resulted in a laterally directed force causing the player to land with the ankle in a an excessive inverted position; and (2) forced plantarflexion when the injured player hit the opponent's foot when attempting to shoot or clear the ball. However, those conclusions only revealed the injury mechanism qualitatively. Although determination of the direct cause of the injury, namely the joint loading, may be difficult based on video analysis (Krosshaug and Bahr, 2005), a recent study on the mechanisms of ACL injuries (Koga et al. 2010) have clearly demonstrated that quantification of the observed kinematics can provide important insight into the mechanism of injury. 
A direct approach to study such injuries is to analyze video sequences of real ankle sprain injury incidents captured during televised sport events. However, it is not possible to use standard biomechanical method to analyse these video sequences (Krosshaug and Bahr, 2005). Krosshaug and Bahr (2005) introduced a Model-Based Image-Matching (MBIM) technique for reconstructing three-dimensional human motion from uncalibrated video sequences, and successfully employed this technique to analyze anterior cruciate ligament injuries (Krosshaug et al., 2007, Koga et al., 2010).

The developed MBIM technique has been validated, but only validated for the hip and knee joints. In order to utilize the MBIM technique to analyze ankle joint motions, it is necessary to first evaluate its validity and reproducibility. Therefore, the purpose of this study was to validate the MBIM technique for estimating ankle joint kinematics in a cadaveric lower limb specimen using bone-pin marker-based motion analysis as the gold standard."

\section{Line 20 \\ "conduct" should be conducting}

Authors' response: That sentence has been removed because of the rearrangement of introduction.

Materials and Methods

\section{Line 36-37}

\section{How were these lengths defined?}

Authors' response: Definitions were added to line 55-60.

"The shank length was defined as the distance between the lateral femoral epidcondyle and lateral malleolus. Shank circumference was defined as the maximum circumference along the shank. Foot length was defined as the anterior-posterior length measurement from the lateral calcaneus to the tip of the long toe; foot width was defined as the maximal medial-lateral distance measured perpendicular to the long axis of the foot."

\section{Lines 40 and 56}

Joint range of motion is never defined. What were the specific ranges that the specimens went through?

\section{Authors' response:}

The range of motion data were added to Table 1 .

The table below summarized the range of motion

\begin{tabular}{|c|c|c|c|c|c|}
\hline & $\begin{array}{l}\text { Plantarflexion } \\
\text { Dorsiflexion - }\end{array}$ & +1 & $\begin{array}{l}\text { Inversion } \\
\text { Eversion - }\end{array}$ & + & $\begin{array}{ll}\text { Internal rotation }+/ \\
\text { External rotation - }\end{array}$ \\
\hline Bare-foot & $-39.7 \rightarrow 67.9$ & & $-48.7 \rightarrow 82.3$ & & $-13.7 \rightarrow 5.9$ \\
\hline Shoe-wearing & $-47.6 \rightarrow 48.4$ & & $-42.5 \rightarrow 61.7$ & & $-11.4 \rightarrow 6.7$ \\
\hline
\end{tabular}




\section{Lines 58-60}

This sentence needs to be corrected for grammar.

Authors' response: The sentence was rewritten.

Line 85-88:

"The video recordings from the four video cameras were analyzed by a video motion analysis system (Ariel Performance Analysis System, USA) which was used to calculate the reflective marker's three-dimensional coordinates."

\section{Lines 67-75}

The methodology in this section needs much greater detail. How many total images (or positions) were analyzed? Were the images randomized? Were the images evaluated at specific angles within the range of motion? Did each researcher view each image, and how many times?

Authors' response: Thank you for your comment. Randomization is not included in the methodology because the images analyzed were in a sequence as a whole. And the whole images sequences included full range of ankle joint motion in $30 \mathrm{~Hz}$. The detailed descriptions were added.

Line 71-72:

"Four video cameras (Casio EX-F1, Tokyo, Japan) were used to record the ankle motion at $30 \mathrm{~Hz}$ with $640 \times 480$ resolutions from different views."

Line 83-85:

"Full-range plantarflexion/dorsiflexion, inversion/eversion and relative circular motion between the two shank and foot segments were performed on the ankle joint with maximal manual effort."

Line 94-114:

"The matchings were performed using the commercially available program $\operatorname{Poser}^{\circledR} 4$ and the Poser ${ }^{\circledR}$ Pro Pack (Curious Labs Inc., Santa Cruz, California, USA). First, models of the surroundings were manually matched to the background for each frame in every camera view, using a key frame and spline interpolation technique, by adjusting the camera calibration parameters (position, orientation and focal length). The surroundings were modeled using points, straight lines, for instance, the boundaries of the mechanical jig. We utilized a skeleton model from Zygote Media Group Inc. (Provo, Utah, USA) for the athlete matching of the leg. The model for lower extremity consisted of 9 rigid segments with a hierarchical structure, using the pelvis as the parent segment. In our study, 5 rigid segments were enough for one side. The pelvis motion was described by three rotational and three translational degrees of freedom. The motion of the remaining segments was then described with three rotational degrees of freedom relative to their parent, e.g., the foot relative to the shank. The matching procedure has been described in detail by Krosshaug and Bahr (2005). Two researchers, A and B, performed the manual skeleton matching process five times on each specimen. Both researchers possessed good human biomechanics knowledge and were trained to implement the MBIM technique by following the same protocol (Figure 2). Because the default ankle joint center of the Zygote skeleton model was not located at the mid-point between the malleoli, the ankle joint centre was adjusted in the Joint Editor Section of the Poser software."

Results 


\section{Line 99}

Again it is important to report the ROM values. If the ROM is 50 and the error is 3 that is acceptable. If the ROM is 6 and the error is 3 then that may not be acceptable.

Authors' response:

The range of motion data were added to Table 1 .

The table below summarized the range of motion

\begin{tabular}{|c|c|c|c|c|c|}
\hline & $\begin{array}{l}\text { Plantarflexion } \\
\text { Dorsiflexion - }\end{array}$ & +1 & $\begin{array}{l}\text { Inversion } \\
\text { Eversion - }\end{array}$ & + & $\begin{array}{l}\text { Internal rotation }+/ \\
\text { External rotation - }\end{array}$ \\
\hline Bare-foot & $-39.7 \rightarrow 67.9$ & & $-48.7 \rightarrow 82.3$ & & $-13.7 \rightarrow 5.9$ \\
\hline Shoe-wearing & $-47.6 \rightarrow 48.4$ & & $-42.5 \rightarrow 61.7$ & & $-11.4 \rightarrow 6.7$ \\
\hline
\end{tabular}

\section{Lines 102 and 107}

Table 2 should not come before Table 1 in the text. Either the table labels or the text should be switched.

Authors' response:

Table 1 and table 2 were inter-changed.

Discussion

\section{Line 122}

What are the skin marker errors compared to? Bone pins?

Authors' response:

That previous study compared the skin markers to bone-pins markers.

Line 156-159:

"Previous studies comparing skin markers compared to bone-pin markers gave RMS error of $4.7^{\circ}$ for plantarflexion/dorsiflexion angle, $4.6^{\circ}$ for inversion/eversion angle and $3.6^{\circ}$ for internal/external rotation angle under slow speed running (Reinschmidt et al., 1997a)."

\section{Line 133}

"is" should be in

Authors' response:

"is" is changed to "in"

Line 168-169:

"Nevertheless, the accuracy of MBIM technique in shoed conditions is still very good."

\section{Line 155}

How does this relate to actual camera images taken during sporting events? Will the data be affected?

Authors' response:

MBIM is the same as the other motion analysis, the accuracy and reliability are affected by the quality of image captures. In our study, 4 separate camera views is likely important for the excellent results that was seen. However, we believe this 
situation is representative for videos that we can obtain from actual videos of injury situations. Although the cameras filmed from a relatively close distance, the picture resolution used here is only apx $15 \%$ of what is found in todays HD broadcasts, widely used in major sports events and soon to be the standard in all TV-broadcasts. Such high quality video footage of injury situations is previously used for analysis of ACL injuries (Koga et al. 2010).

\section{Line 173}

"form" should be from

Authors' response:

The sentence has been removed. Thanks!

\section{Lines 174-175}

\section{This sentence needs to be corrected for grammar.}

Authors' response:

The sentence was rewritten as below.

Line 209-211

"MBIM motion analysis technique may potentially be developed into a sophisticated video analysis for research or clinical uses, such as the mechanisms of injuries captured on tape." 
Reviewer \#3: This is an interesting study that validated the MBIM technique for the ankle. Looking at the literature, it appears that the subject is novel and original. It is questionable, however, if Gait and Posture readers are indeed the correct forum for this type of study: the experiment itself would be more relevant to a biomechanics journal, whilst the clinical application would be mostly in the field of sport medicine.

The paper is difficult to read and follow for several different reasons:

1. the language is poor and the text would require editing by a native English speaker.

Authors' response:

We thank you for your advice. The revised manuscript has now been proof-read by a native English researcher at our university. We hope this has made the manuscript acceptable.

2. the Authors assume that the reader is familiar with the MBIM technique and give insufficient detail about their methodology.

3. The experiment should be described in enough detail to make it reproducible in a different lab. For example it is unclear (despite the photograph in figure 1) how the Authors managed to fit a basketball shoe around the bone pins.

Authors' response to Question 2 and 3:

A hole on the lateral posterior side of the shoe was prepared for the penetration of bone-pins, given that there is no interference between the bone-pins and shoes. The detailed descriptions of MBIM procedures were added as below.

Line 67-70:

"Figure 1 showed the bone-pin makers on cadavers with two testing conditions, bare-foot and shoed. A hole on the lateral posterior side of the shoe was prepared for the penetration of bone-pins, given that there is no interference between the bone-pins and shoes."

Line 94-108:

"The matchings were performed using the commercially available program $\operatorname{Poser}^{\circledR} 4$ and the Poser ${ }^{\circledR}$ Pro Pack (Curious Labs Inc., Santa Cruz, California, USA). First, models of the surroundings were manually matched to the background for each frame in every camera view, using a key frame and spline interpolation technique, by adjusting the camera calibration parameters (position, orientation and focal length). The surroundings were modeled using points, straight lines, for instance, the boundaries of the mechanical jig. We utilized a skeleton model from Zygote Media Group Inc. (Provo, Utah, USA) for the athlete matching of the leg. The model for lower extremity consisted of 9 rigid segments with a hierarchical structure, using the pelvis as the parent segment. In our study, 5 rigid segments were enough for one side. The pelvis motion was described by three rotational and three translational degrees of freedom. The motion of the remaining segments was then described with three rotational degrees of freedom relative to their parent, e.g., the foot relative to the shank. The matching procedure has been described in detail by Krosshaug and Bahr (2005)." 
I note that the Achilles tendon and other soft tissues were divided to allow more movement around the ankle. I believe the validation should have been undertaken within the constraints of the range of motion allowed by the real structures around the ankle. Increasing the range of motion could have influenced the validity testing.

Authors' response: The reason why we wanted to study large ankle ranges of motions was our desire to later utilize the MBIM technique to analyze injury case in which extreme joint orientation can be present. So, it is essential to validate the MBIM technique outside the normal range of motion. In this case, we were very carefully in tearing the soft tissues in order avoid changing the soft structures more than necessary.

There is indeed interesting and useful information in the present study and it certainly reflects quite a lot of serious work. It does however require major re-writing before it is publishable. It would be up to the Editors to decide if $\mathrm{G} \& \mathrm{P}$ is the right journal for this paper. 


\begin{tabular}{|c|c|c|c|}
\hline Title & \multicolumn{3}{|c|}{ An ankle joint model-based image-matching motion analysis technique } \\
\hline Article type & \multicolumn{3}{|l|}{ Original Paper } \\
\hline Authors & \multicolumn{3}{|c|}{$\begin{array}{l}\text { Kam-Ming MOK }{ }^{1,2}, \text { Daniel Tik-Pui FONG } \\
\text { Aaron See-Long } \text { HUNG }^{1,2} \text {, Pron KROSSHAUG } \\
\text {, } \\
\text { CHAN }^{1,2}\end{array}$} \\
\hline Affiliations & \multicolumn{3}{|c|}{$\begin{array}{l}{ }^{1} \text { Department of Orthopaedics and Traumatology, Prince of Wales } \\
\text { Hospital, Faculty of Medicine, The Chinese University of Hong Kong, } \\
\text { Hong Kong, China } \\
{ }^{2} \text { The Hong Kong Jockey Club Sports Medicine and Health Sciences } \\
\text { Centre, Faculty of Medicine, The Chinese University of Hong Kong, } \\
\text { Hong Kong, China } \\
{ }^{3} \text { Oslo Sports Trauma Research Center, Norwegian School of Sport } \\
\text { Sciences, Ullevaal Stadion, Oslo, Norway } \\
{ }^{4} \text { Department of Orthopaedics and Traumatology, Alice Ho Miu Ling } \\
\text { Nethersole Hospital, Hong Kong, China }{ }^{4}\end{array}$} \\
\hline Keywords & \multicolumn{3}{|c|}{ video analysis, ankle joint motion, injury biomechanics } \\
\hline Total words & 2860 & Words in abstract & 127 \\
\hline \multicolumn{4}{|c|}{ Correspondence author during review process: Kam-Ming MOK } \\
\hline Telephone & (852) 26364171 & Facsimile & $(852) 26463020$ \\
\hline E-mail & \multicolumn{3}{|c|}{ kmmok@ort.cuhk.edu.hk } \\
\hline \multicolumn{4}{|c|}{ Correspondence author if paper is accepted: Daniel Tik-Pui FONG } \\
\hline Telephone & (852) 26323535 & Facsimile & $(852) 26463020$ \\
\hline E-mail & \multicolumn{3}{|c|}{ dfong@ ort.cuhk.edu.hk } \\
\hline
\end{tabular}




\section{ABSTRACT}

2 This study presented a model-based image-matching (MBIM) motion analysis

3 technique for ankle joint kinematic measurement. Five cadaveric below-hip

4 specimens were manipulated through a full range of ankle joint motions in bare-foot

5 and shoed conditions. The ankle motions were analyzed by bone-pin marker-based

6 motion analysis and MBIM motion analysis techniques respectively. The root mean

7 square errors of all angles of motion were less than 3 degrees. The average Intraclass

8 Correlation Coefficients (ICCs) for the intra-rater reliability were greater than 0.928

9 and the average ICCs for the inter-rater reliability were greater than 0.948 for all

10 ranges of motion. Excellent validity, intra-rater reliability and inter-rater reliability

11 were achieved for the MBIM technique in both bare-foot and shoed conditions. The

12 MBIM technique can therefore provide good estimates of ankle joint kinematics.

\section{INTRODUCTION}

15 Ankle ligamentous sprain is one of the most common injuries encountered in sports

16 (Fong et al., 2007; Fong et al., 2009a). A precise description of the injury situation is a

17 key component to understanding the aetiology and injury mechanism (Bahr and

18 Krosshaug, 2005). The injury mechanisms of ankle ligamentous sprain have been

19 described as a combined inversion and internal rotation of the ankle joint (Safran et al.,

20 1991), or plantarflexion with the subtalar joint adducting and inverting (Vitale \&

21 Fallat, 1988). Fong et al. (2009b) reported the ankle joint kinematics from a single

22 accidental ankle supination sprain case under skin-marker motion analysis, the finding

23 is that dorsiflexion instead of plantarflexion was found at injury. A study analyzed the

24 ankle supination sprain injuries using video analysis, Andersen et al. (2004) reported

25 two major injury mechanisms as: (1) impact by opponent on the medial aspect of the 
leg just before or at foot strike, which resulted in a laterally directed force causing the player to land with the ankle in a an excessive inverted position; and (2) forced plantarflexion when the injured player hit the opponent's foot when attempting to shoot or clear the ball. However, those conclusions only revealed the injury mechanism qualitatively. Although determination of the direct cause of the injury, namely the joint loading, may be difficult based on video analysis (Krosshaug and Bahr, 2005), a recent study on the mechanisms of ACL injuries (Koga et al. 2010) have clearly demonstrated that quantification of the observed kinematics can provide important insight into the mechanism of injury.

A direct approach to study such injuries is to analyze video sequences of real ankle sprain injury incidents captured during televised sport events. However, it is not possible to use standard biomechanical method to analyse these video sequences (Krosshaug and Bahr, 2005). Krosshaug and Bahr (2005) introduced a Model-Based Image-Matching (MBIM) technique for reconstructing three-dimensional human motion from uncalibrated video sequences, and successfully employed this technique to analyze anterior cruciate ligament injuries (Krosshaug et al., 2007, Koga et al., 2010).

The developed MBIM technique has been validated, but only validated for the hip and knee joints. In order to utilize the MBIM technique to analyze ankle joint motions, it is necessary to first evaluate its validity and reproducibility. Therefore, the purpose of this study was to validate the MBIM technique for estimating ankle joint kinematics in a cadaveric lower limb specimen using bone-pin marker-based motion analysis as the gold standard. 


\section{Experimental setup}

52 Five cadaveric below-hip specimens $($ shank length $=32.4 \pm 1.9 \mathrm{~cm}$, shank

53 circumference $=24.6 \pm 1.4 \mathrm{~cm}$, foot length $=22.5 \pm 0.7 \mathrm{~cm}$, foot width $=8.2 \pm 0.6 \mathrm{~cm})$

54 were prepared for testing. The shank length was defined as the distance between the

55 lateral femoral epidcondyle and lateral malleolus. Shank circumference was defined 56 as the maximum circumference along the shank. Foot length was defined as the 57 anterior-posterior length measurement from the lateral calcaneus to the tip of the long 58 toe; foot width was defined as the maximal medial-lateral distance measured 59 perpendicular to the long axis of the foot. These anthropometrical measurements were 60 used to customize the skeleton model used in the Model-Based Image-Matching 61 technique. The Achilles tendon and surrounding soft tissues around the ankle joint were dissected to increase joint range of motion, given that basic structure was intact.

\section{Bone-pin marker based video motion analysis}

Hofmann II external fixation 5.0mm bone-pins (Stryker, USA) with triads of reflective markers were drilled into the posterolateral side of the calcaneus and into the tibia through the lateral tibial condyle (Reinschmidt et al., 1997a). Figure 1 showed the bone-pin makers on cadavers with two testing conditions, bare-foot and shoed. A hole on the lateral posterior side of the shoe was prepared for the penetration

69 of bone-pins, given that there is no interference between the bone-pins and shoes. 70 Four video cameras (Casio EX-F1, Tokyo, Japan) were used to record the ankle 71 motion at $30 \mathrm{~Hz}$ with $640 \times 480$ resolutions from different views. A static calibration trial in the anatomical position served as the offset position to determine the segment

73 embedded axes of the shank and foot segment. The foot coordinate system was

74 aligned with the Laboratory Coordinate System (LCS) (Reinschmidt et al., 1997b).

75 Reflective skin markers were attached to the lateral femoral epicondyle, medial 
76 femoral epicondyle, lateral malleolus and medial malleolus to define knee and ankle

77 joint centers (Wu et al., 2002). These markers were removed after the static

78 calibration. The line connecting the knee joint centre and the ankle joint centre was

79 defined as the longitudinal axis of the shank segment (X1). The anterior-posterior axis

80 of the shank segment (X2) was the cross product between X1 and the line joining the

81 lateral femoral epicondyle and medial femoral epicondyle. The medial-lateral axis of 82 the shank segment was the cross product of X1 and X2. Full-range 83 plantarflexion/dorsiflexion, inversion/eversion and relative circular motion between 84 the two shank and foot segments were performed manually on the ankle joint. The 85 video recordings from the four video cameras were analyzed by a video motion 86 analysis system (Ariel Performance Analysis System, USA) which was used to 87 calculate the reflective marker's three-dimensional coordinates. A singular value 88 decomposition method was employed to calculate the transformation from triad 89 reference frame to anatomical shank and foot reference frame (Sodervist and Wedin, 90 1993). Joint kinematics were resolved by the Joint Coordinate System (JCS) method

91 (Grood and Suntay, 1983).

\section{Model-Based Image-Matching motion analysis}

93 The videos were analyzed using the MBIM technique (Figure 3). The matchings were 94 performed using the commercially available program Poser $^{\circledR} 4$ and the Poser $^{\circledR}$ Pro 95 Pack (Curious Labs Inc., Santa Cruz, California, USA). First, models of the 96 surroundings were manually matched to the background for each frame in every 97 camera view, using a key frame and spline interpolation technique, by adjusting the 98 camera calibration parameters (position, orientation and focal length). The 99 surroundings were modeled using points, straight lines, for instance, the boundaries of 100 the mechanical jig. We utilized a skeleton model from Zygote Media Group Inc. 
101 (Provo, Utah, USA) for the athlete matching of the leg. The model for lower extremity consisted of 9 rigid segments with a hierarchical structure, using the pelvis as the

103 parent segment. In our study, 5 rigid segments were enough for one side. The pelvis

104 motion was described by three rotational and three translational degrees of freedom.

105 The motion of the remaining segments was then described with three rotational

106 degrees of freedom relative to their parent, e.g., the foot relative to the shank. The 107 matching procedure has been described in detail by Krosshaug and Bahr (2005). Two

108 researchers, $\mathrm{A}$ and $\mathrm{B}$, performed the manual skeleton matching process five times on

109 each specimen. Both researchers possessed good human biomechanics knowledge and

110 were trained to implement the MBIM technique by following the same protocol

111 (Figure 2). Because the default ankle joint center of the Zygote skeleton model was

112 not located at the mid-point between the malleoli, the ankle joint centre was adjusted

113 in the Joint Editor Section of the Poser software. The centre of ankle joint were preset

114 as right side [-0.045 $0.030-0.008]$ and left ankle side [0.045 $0.030-0.008]$ according

115 to the joint centre definition in ISB recommendation (Wu et al., 2002). After the initial

116 matching was completed, the motions of the skeleton model were reassessed and

117 adjusted frame by frame to ensure a smoothed motion.

\section{Statistical analysis}

119 The differences between bone-pin marker-based motion analysis and MBIM

120 technique were quantified using Root Mean Square (RMS) error. Bivariate Pearson

121 correlations were calculated to compare the similarity of the trends between the two

122 techniques. Intra-rater reliability and inter-rater reliability within the MBIM technique

123 were assessed using Intraclass Correlation Coefficients (ICCs). Since the MBIM

124 technique provide continuous joint angle time histories, ICCs with two-way mixed

125 model average measures were calculated to evaluate reliability (Hopkins, 2000). 
126 Fleiss (1986) suggested that an ICC coefficient of $>0.75$ was considered as evidence

127 of good agreement. However, in the present study, we defined that an ICC coefficient 128 of $>0.90$ was required to achieve excellent reliability.

\section{$130 \quad$ RESULTS}

$131 \quad$ Validity

132 In both testing conditions, the RMS errors were less than three degrees for all angles 133 of motion (plantar/dorsiflexion, inversion/eversion, internal/external rotation). The 134 measurement difference, standard deviation of difference, 95\% limits of agreement 135 and related statistical results were reported in table 1. The Pearson's correlations were

136 higher than 0.946 for all angles of motion and conditions. In general, the MBIM 137 technique achieved excellent accuracy and correlation with the results from the 138 bone-pin marker-based motion analysis.

\section{Intra-rater reliability}

140 Results of ICC coefficients on three angles of motion were shown in table 2. In both

141 bare-foot and shoed conditions, the ICC coefficients for intra-rater reliability 142 demonstrated excellent correlation (ICC coefficient $>0.955$ ) for all angles of motion.

143 Intra-rater reliability was considered to have been achieved as all ICC coefficients 144 were greater than 0.950 , and the analysis was reproducible from a single researcher.

\section{Inter-rater reliability}

146 Results of ICC coefficients on three angle of motion were shown in table 3 . In both 147 testing conditions, the ICC coefficients for inter-rater reliability demonstrated 148 excellent correlation (ICC coefficient >0.952) for angles of motion between two 149 investigators. Inter-rater reliability was considered to have been achieved as all ICC 150 coefficients were greater than 0.90 , and the analysis was reproducible for different 
researchers.

\section{DISCUSSION}

154 Skin-marker based motion analysis is the most common present approach to

155 investigate joint kinematics. Previous studies comparing skin markers compared to

156 bone-pin markers gave RMS error of $4.7^{\circ}$ for plantarflexion/dorsiflexion angle, $4.6^{\circ}$

157 for inversion/eversion angle and $3.6^{\circ}$ for internal/external rotation angle under slow

158 speed running (Reinschmidt et al., 1997a). For MBIM motion analysis technique, the

159 RMS errors of the three angles of motion were less than $3^{\circ}$ for the entire testing

160 motion (Table 2), the expected improvement in accuracy using bone pins was evident,

161 although a direct comparison was not possible since neither in the running or ankle

162 manipulation studies were both recorded concurrently. In our study, bare-foot and

163 shoed conditions were also tested. Basketball shoes was chosen because basketball

164 shoes had high tops which covered the whole ankle joint, and this made the most

165 difficult situation for the skeleton matching process. By visual inspection, there was

166 shear movement between the foot and shoe, the underlying movement of foot segment

167 was hidden. Nevertheless, the accuracy of MBIM technique in shoed conditions is

168 still very good. Regarding the reliability of the MBIM technique, the average ICC

169 coefficients for the intra-rater reliability were greater than 0.928 for all ranges of

170 motion and the average ICC coefficients for the inter-rater reliability were greater than

171 0.948. These results implied that different trained researchers can produce the same

172 results with excellent reliability.

174 A detailed protocol for the matching is suggested in this study, which we believe is

175 crucial for the excellent results. During the skeleton matching process, researchers 
176 should be carefully in identifying the longitudinal axis orientations of the shank and

177 the foot segments. Inversion/eversion, it was highly dependant on the orientation of

178 the foot segment. The foot segment could be regarded as a rectangular board. The

179 orientation of the plantar foot would be key information to match the foot skeleton on

180 the video images. Using the top view camera and front view camera in Poser, the

181 detailed orientation of the foot segment could be seen and further fine tuning was

182 possible. In the previous validation study of Krosshaug and Bahr (2005) a relatively

183 large discrepancy in internal/external rotation of the knee joint was obtained between

184 the Poser method and the reflective marker based method. This was identified to

185 originate form the thigh segment, likely due to soft tissue artifacts of the thigh relative

186 to the underlying bone (Krosshaug \& Bahr, 2005). Similarly, the shank was

187 comparably difficult to be perfectly matched. In the matching of the tibia model on

188 the images, the patellar position and the anterior edge of the shank were the decisive

189 landmarks to define the internal rotation orientation of the shank. Those two

190 anatomical landmarks were chosen because the underlying soft tissue was relatively

191 thin, and they could precisely reflect the rotation orientation of the tibia. Lastly,

192 researchers were suggested to reassess the motion of the skeleton model for the whole

193 video and adjusted frame by frame to ensure a smooth matched motion.

194 The MBIM motion analysis technique is a novel approach to reconstruct the

195 three-dimensional kinematics from uncalibrated video sequences, however the authors

196 would like to point out several directions for the MBIM technique to be further

197 developed. Firstly, more than four commercial softwares were employed in the whole

198 analysis. It would be more user-friendly and time-effective if an all-in-one software

199 was developed. Secondly, the skeleton matching process was extremely

200 time-consuming to the researcher. The process could be more time-saving if camera 
201 position estimation and edge detection technique were implemented (Oe et al., 2005).

202 The camera position estimation technique could help matching the virtual

203 environment in a more precise and faster manner, and the edge detection technique

204 could objectively outline the segment boundary for skeleton matching. However, this

205 kind of development was currently not possible on the MBIM motion analysis

206 technique because of the dependence on commercial softwares. The kinematics can be

207 further analyzed by to figure out the internal stress and liagmentous tension (Chao et

208 al., 2007). MBIM motion analysis technique may potentially be developed into a

209 sophisticated video analysis for research or clinical uses, such as the mechanisms of

210 injuries captured on tape.

\section{CONCLUSION}

213 Excellent validity, intra-rater reliability and inter-rater reliability were achieved for the 214 MBIM technique in both bare-foot and shoed conditions. The MBIM motion analysis 215 technique can therefore provide excellent estimates of ankle joint kinematics.

\section{ACKNOWLEDGEMENT}

218 This research project was made possible by resources donated by The Hong Kong 219 Jockey Club Charities Trust.

\section{REFERENCES}

222 1. Andersen TE, Floerenes TW, Arnason A, Bahr R. Video analysis of the mechanisms for ankle injuries in football. American Journal of Sports Medicine 2004, 32(Suppl): S69-S79.

2. Bahr R, \& Krosshaug T. Understanding injury mechanisms: A key component of preventing injuries in sports. British Journal of Sports Medicine 2005, 39(6): 324-329. 
3. Chan KM, Fong DTP, Hong Y, Yung PSH, Lui PPY. Orthopaedic sport biomechanics - a new paradigm. Clinical Biomechanics 2008, 23(1 Supp): 21-30.

4. Chao EYS, Armiger RS, Yoshida H, Lim J, Haraguchi N. Virtual interactive musculoskeletal system (VIMS) in orthopaedic research, education and clinical patient care. Journal of Orthopaedic Surgery and Research 2007, 2:2.

5. Fleiss JL (Ed), (1986) The design and analysis of clinical experiments New York, John Wiley \& Sons.

6. Fong DTP, Hong Y, Chan LK, Yung PSH, Chan KM. A systematic review on ankle injury and ankle sprain in sports. Sports Medicine 2007; 37(1): 73-94.

7. Fong DTP, Chan YY, Mok KM, Yung PSH, Chan KM. Understanding acute ankle ligamentous sprain injury in sports. Sports Medicine, Arthroscopy, Rehabilitation, Therapy and Technology 2009a; 1: 14.

8. Fong DTP, Hong Y, Shima Y, Krosshuag T, Yung PSH, Chan KM. Biomechanics of supination ankle sprain: a case report of an accidental injury event in the laboratory. American Journal of Sports Medicine 2009b; 37(4): 822-827.

9. Grood E S \& Suntay W J. A joint coordinate system for the clinical description of three-dimensional motions: application to the knee. Journal of Biomechanical Engineering 1983; 105(2): 136-144.

10. Hopkins WG. Measures of Reliability in Sports Medicine and Science. Sports Medicine 2000; 30(1): 1-15.

11. Krosshaug $\mathrm{T} \& \mathrm{Bahr} \mathrm{R}$. A model-based image-matching technique for three-dimensional reconstruction of human motion from uncalibrated video sequences. Journal of Biomechanics 2005; 38(4): 919-929.

12. Krosshaug T, Slauterbeck JR, Engebretsen L, Bahr, R. Biomechanical analysis of anterior cruciate ligament injury mechanisms: three-dimensional motion reconstruction from video sequences. Scandinavian Journal of Medicine and Science in Sports 2007; 17(5): 508-519.

13. Koga H, Nakamae A, Shima Y, Iwasa J, Myklebust G, Engretsen L, Bahr R, Krosshaug T. Mechanisms for noncontact anterior cruciate ligament injuries. American Journal of Sports Medicine 2010; 35(11):2218-2225.

14. Oe M, Sato T, Yokoya N. Estimating Camera Position and Posture by Using Feature Landmark Database: Lectures Notes in Computer Sciences Heideberg, Springer Berlin 2005: 171-181. 
15. Reinschmidt C, van Den Bogert AJ, Murphy N, Lundberg A, Nigg BM. Tibiocalcaneal motion during running, measured with external and bone markers. Clinical Biomechanics 1997a; 12(1): 8-16.

16. Reinschmidt C, van Den Bogert AJ, Nigg BM, Murphy N, Lundberg A. Effect of skin movement on the analysis of skeletal knee joint motion during running. Journal of Biomechanics 1997b; 30(7): 729-732.

19. Vitale TD, Fallat LM. Lateral ankle sprains: evaluation and treatment. Journal of Foot Surgery 1988, 27(3): 248-258.

20. Wu G, Siegler S, Allard P, Kirtley C, Leardini A, Rosenbaum D, Whittle M, D'Lima DD, Cristofolini L, Witte H, Schmid O, Stokes I. ISB recommendation on definitions of joint coordinate system of various joints for the reporting of human joint motion--part I: ankle, hip, and spine. Journal of Biomechanics 2002; 35(4): 543-548.

21. Zinder SM, Granata KP, Padua DA, Gansneder BM. Validity and reliability of a new in vivo ankle stiffness measurement device. Journal of Biomechanics 2007; $0(2): 463-467$.

289 Figure 2. An example of finished skeleton matching using MBIM motion analysis 290 technique, skeleton model on video images

291 Figure 3. Protocol of the ankle joint model-based image-matching motion analysis 292 technique 


\section{TABLES}

Table 1. Accuracy and Correlation for all angles of motion in two testing conditions

\begin{tabular}{|c|c|c|}
\hline & Bare-foot & Shoed \\
\hline \multicolumn{3}{|l|}{ Plantarflexion + / Dorsiflexion - } \\
\hline Root mean square (rms) error & 2.08 & 2.36 \\
\hline Mean difference (d) & -0.27 & 0.20 \\
\hline S.D. of difference & 2.05 & 2.47 \\
\hline $95 \%$ limits of agreement & $-4.29 \rightarrow 3.75$ & $-4.64 \rightarrow 5.04$ \\
\hline Pearson's correlation $(\mathrm{R})$ & 0.996 & 0.985 \\
\hline $\mathrm{R}$ Square $\left(\mathrm{R}^{2}\right)$ & 0.991 & 0.971 \\
\hline Average range of motion (deg) & $-39.7 \rightarrow 67.9$ & $-47.6 \rightarrow 48.4$ \\
\hline \multicolumn{3}{|l|}{ Inversion + / Eversion - } \\
\hline Root mean square (rms) error & 1.99 & 2.95 \\
\hline Mean difference (d) & 0.29 & 0.82 \\
\hline S.D. of difference & 2.09 & 2.97 \\
\hline $95 \%$ limits of agreement & $-3.81 \rightarrow 4.39$ & $-5.00 \rightarrow 6.64$ \\
\hline Pearson's correlation (R) & 0.996 & 0.998 \\
\hline $\mathrm{R}$ Square $\left(\mathrm{R}^{2}\right)$ & 0.992 & 0.996 \\
\hline Average range of motion (deg) & $-48.7 \rightarrow 82.3$ & $-42.5 \rightarrow 61.7$ \\
\hline \multicolumn{3}{|l|}{ Internal rotation + / External rotation - } \\
\hline Root mean square (rms) error & 2.01 & 2.20 \\
\hline Mean difference (d) & -0.29 & 0.40 \\
\hline S.D. of difference & 1.75 & 2.87 \\
\hline $95 \%$ limits of agreement & $-3.72 \rightarrow 3.14$ & $-5.23 \rightarrow 6.03$ \\
\hline Pearson's correlation (R) & 0.952 & 0.946 \\
\hline $\mathrm{R}$ Square $\left(\mathrm{R}^{2}\right)$ & 0.907 & 0.894 \\
\hline Average range of motion (deg) & $-13.7 \rightarrow 5.9$ & $-11.4 \rightarrow 6.7$ \\
\hline
\end{tabular}


Table 2. Intra-rater Reliability (Intra-Class Correlation)

\begin{tabular}{ccccccc}
\hline & Plantarflexion/dorsiflexion & \multicolumn{2}{c}{ Inversion/eversion } & \multicolumn{2}{c}{ Internal/external rotation } \\
\hline Researcher & $\mathrm{A}$ & $\mathrm{B}$ & $\mathrm{A}$ & $\mathrm{B}$ & $\mathrm{A}$ & $\mathrm{B}$ \\
\hline Bare-foot & & & & & \\
Leg 1 & 0.999 & 0.998 & 0.997 & 0.993 & 0.957 & 0.968 \\
Leg 2 & 0.997 & 0.999 & 0.999 & 0.999 & 0.991 & 0.987 \\
Leg 3 & 0.997 & 0.996 & 0.992 & 0.995 & 0.986 & 0.983 \\
Leg 4 & 0.998 & 0.999 & 0.997 & 0.999 & 0.978 & 0.981 \\
Leg 5 & 0.992 & 0.998 & 0.999 & 0.999 & 0.971 & 0.958 \\
Average & 0.997 & 0.998 & 0.997 & 0.997 & 0.977 & 0.975 \\
Shoe-wearing & & & & & 0.979 \\
Leg 1 & 0.997 & 0.999 & 0.994 & 0.997 & 0.928 & 0.945 \\
Leg 2 & 0.990 & 0.997 & 0.997 & 0.987 & 0.940 & 0.987 \\
Leg 3 & 0.994 & 0.994 & 0.998 & 0.998 & 0.980 & 0.974 \\
Leg 4 & 0.996 & 0.994 & 0.996 & 0.997 & 0.976 & 0.980 \\
Leg 5 & 0.995 & 0.995 & 0.995 & 0.997 & 0.953 & 0.950 \\
Average & 0.994 & 0.996 & 0.996 & 0.995 & 0.955 & 0.967 \\
\hline
\end{tabular}

Table 3. Inter-rater Reliability (Intra-Class Correlation)

\begin{tabular}{cccc}
\hline Condition & Plantarflexion/dorsiflexion & Inversion/eversion & Internal/external rotation \\
\hline Bare-foot & 0.996 & 0.994 & 0.952 \\
Shoe-wearing & 0.993 & 0.997 & 0.948 \\
\hline
\end{tabular}


Click here to download high resolution image

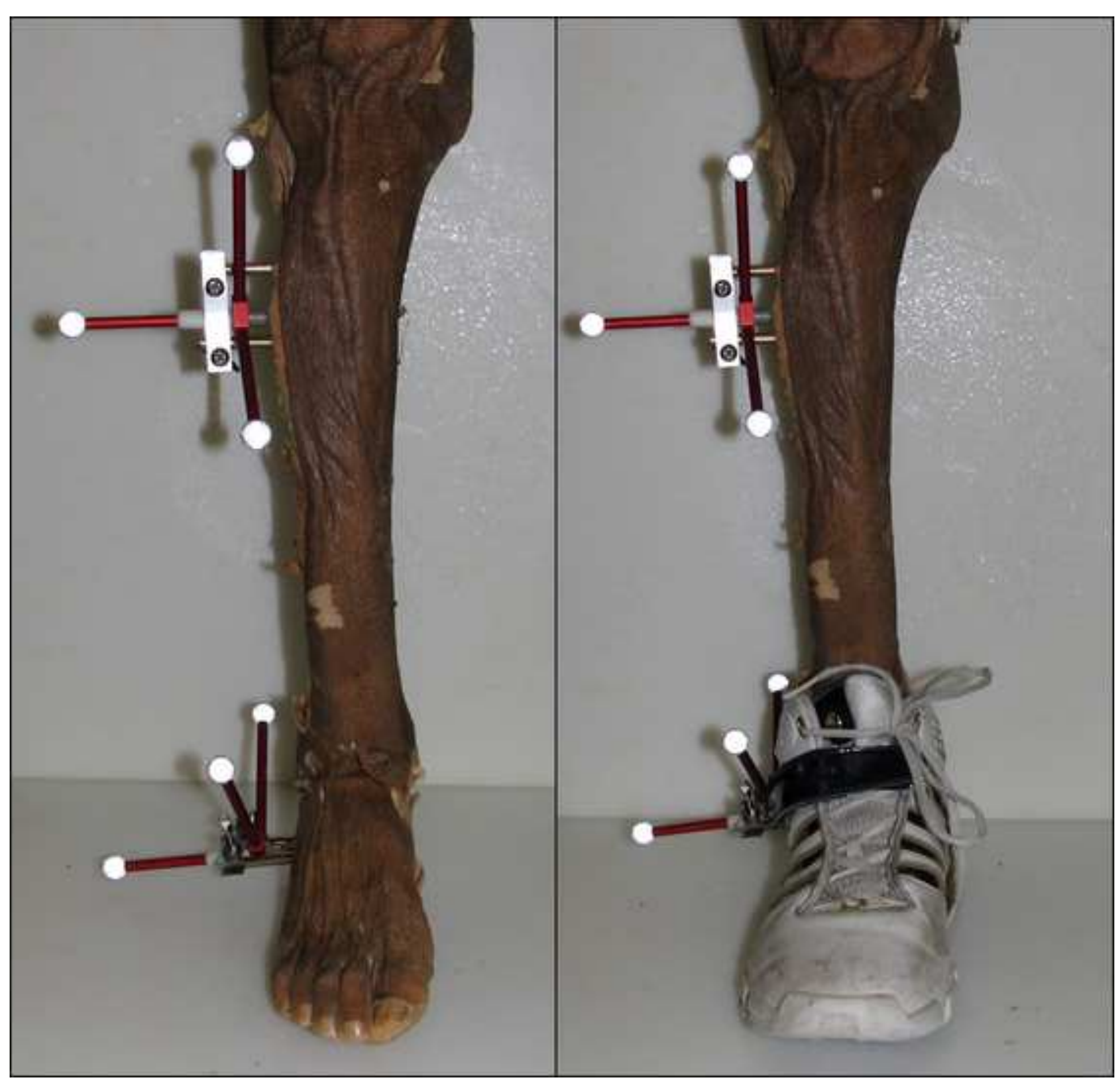




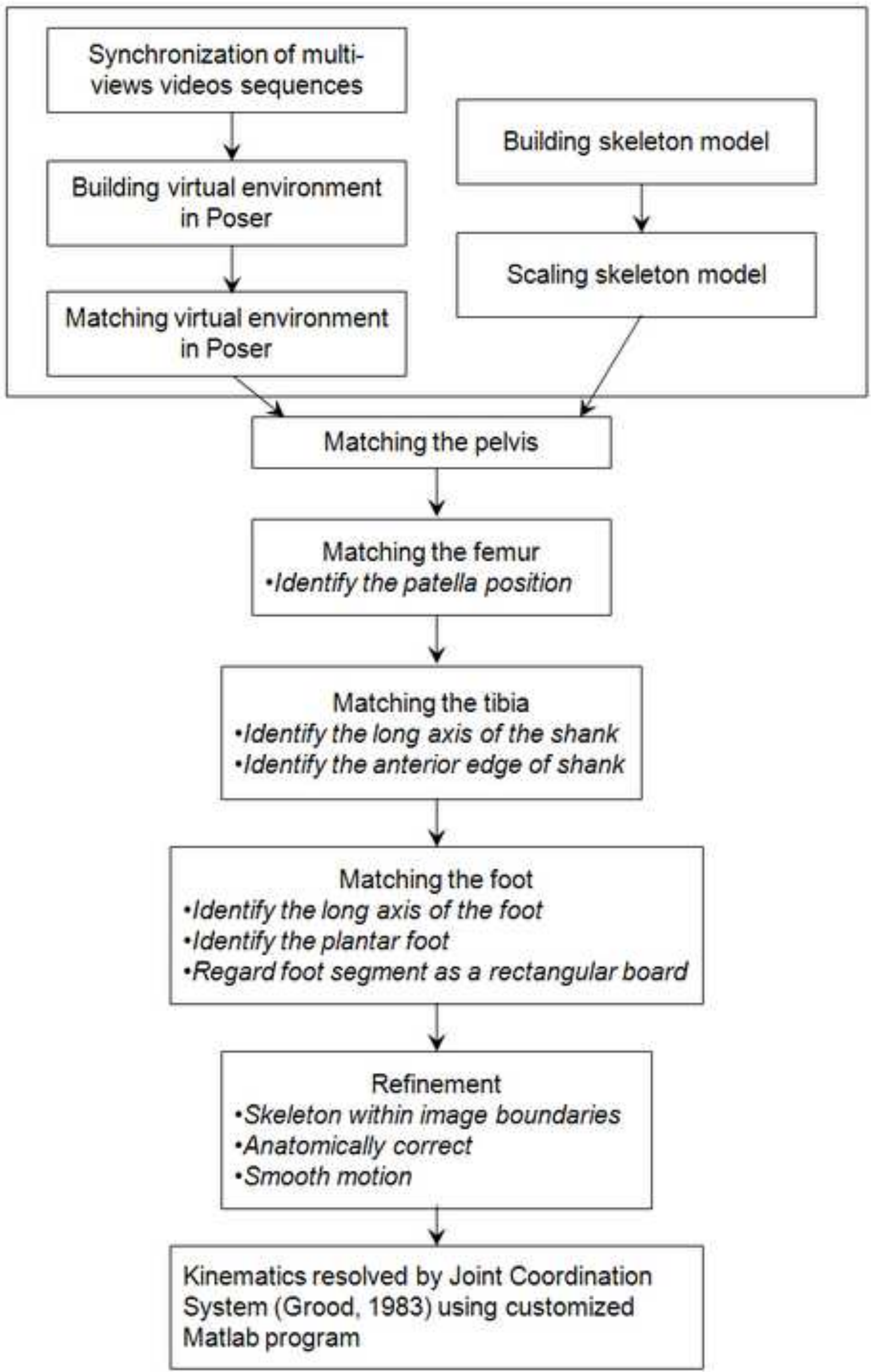




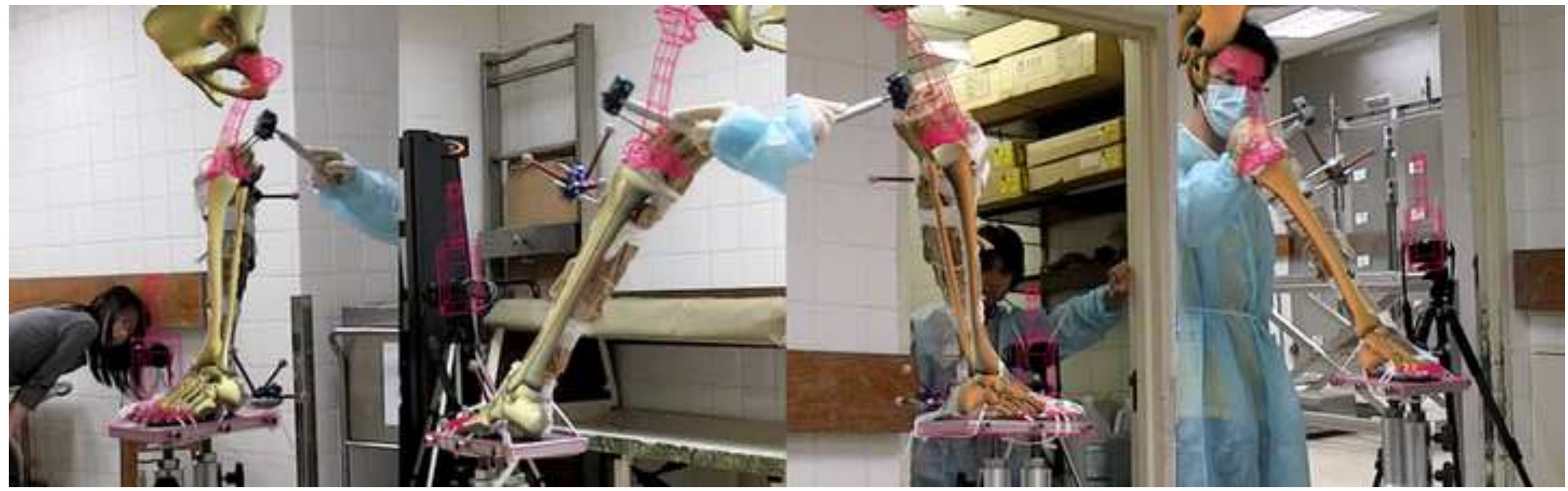

